\title{
Generación de empresas derivadas de base tecnológica (spin offs), a partir de los resultados de I+D+i de los grupos de investigación de la Universidad del Cauca, Colombia
}

Andrés J. Castrillón-Muñoz ${ }^{(1)}$, Alfonso Infante-Moro(2), Alexander Zúñiga-Collazos ${ }^{(3)}$ y Francisco J. Martínez-López ${ }^{(2)}$ (1) Facultad de Ciencias Contables, Económicas y administrativas, Grupo de Inv. en Desarrollo Turístico y Regional, Dpto. de Ciencias del Turismo, Universidad del Cauca, Campus Universitario de Tulcán, Popayán, Cauca - Colombia. (e-mail: andresj9@unicauca.edu.co)

(2) Universidad de Huelva, Grupo de Investigación en Tecnologías de la Información y la Comunicación de la Empresa (GITICE), Campus de "La Merced", Plaza de la Merced, 11. 21.071, Huelva - España. (e-mail: alfonso.infante@uhu.es; francis@uhu.es)

(3) Facultad de Ciencias Económicas, Grupo de Investigación GEOS, Universidad de San Buenaventura, Cali, Valle Colombia. (e-mail: azuniga01@usbcali.edu.co)

Recibido Jun. 14, 2019; Aceptado Ago. 6, 2019; Versión final Oct. 8, 2019, Publicado Feb. 2020

\section{Resumen}

El presente trabajo pretende evidenciar las capacidades que la Universidad del Cauca en Colombia (UNICAUCA), una institución pública nacional de Educación Superior, tendría para convertirse en el mediano plazo en una universidad emprendedora en el marco de un ecosistema de emprendimiento e innovación. Se realiza una evaluación de la literatura científica pertinente, se analizan de referentes legales relacionados con la temática y se obtiene información a través de un censo aplicado a los 64 grupos de investigación de UNICAUCA reconocidos por el Departamento Administrativo de Ciencia, Tecnología e Innovación de COLCIENCIAS en 2017. Los resultados sugieren, entre otras consideraciones relevantes, que el $89 \%$ de ellos, en razón de su perfil y de sus capacidades, estaría en condiciones de desarrollar o de hacer parte de proyectos de base tecnológica tipo Spin Off.

\section{Generation of technological-based spin offs based on I+D+i results from the research groups of the University of Cauca Colombia}

\begin{abstract}
This paper aims to demonstrate the capabilities that the Universidad del Cauca in Colombia (UNICAUCA), a public national institution of Higher Education, would have to become, in the medium term, an entrepreneurial university within the framework of an ecosystem of entrepreneurship and innovation. Relevant scientific literature is explored, legal references related to the subject are analyzed, and information is obtained from a census applied to the 64 research groups recognized by the Administrative Department of Science, Technology and Innovation of COLCIENCIAS in 2017. Results suggest, among other relevant considerations, that $89 \%$ of them, because of their profile and abilities, would be able to develop or be part of Spin Off technology-based projects.
\end{abstract}




\section{INTRODUCCIÓN}

El presente documento pone en evidencia cómo la Universidad del Cauca, República de Colombia, en tanto institución de educación superior pública del orden nacional estaría en condiciones, en el mediano plazo, de articularse en debida forma con organismos del Estado y con empresas externas mediante la creación y posterior consolidación de un modelo para el despliegue de iniciativas de base tecnológica tipo Spin Off, obrando como una universidad emprendedora en el marco de un ecosistema apropiado, el cual, según Hernández y González (2017), citando a Fetters et al (2010) opera cuando confluyen entornos organizacionales, académicos, sociales, políticos y económicos con el objetivo de fundar empresas, en consonancia con los lineamientos de la tercera misión que las Instituciones de Educación Superior deben acometer en tal sentido.

Lo anterior implica considerar la evolución que ha experimentado la universidad desde su aparición como tal y la manera como se ha ido integrando históricamente a las dinámicas de un entorno cada vez más complejo. Nunez y Machado (2018), referenciado a Haskins, (2007), Durkheim, (1995), Giordani, (1982) y Castanho, (2000), plantean que es solo a partir de los Siglos X y XI cuando podría asumirse la existencia de una estructura similar a la que hoy se conoce como universidad, ubicando sus orígenes en Bolonia (1088) y París (1170), caracterizadas por la práctica de la escolástica, propia de las escuelas monásticas cristianas, cuyos objetivos apuntaban más a salvaguardar los valores y creencias de su época que a intereses de mayor espectro. Según estos autores, es en Alemania, a comienzos del S. XIX, (1809-1810) con la creación de la Universidad de Berlín, cuando se institucionaliza el concepto de investigación con Wilhelm von Humboldt, contribuyendo al proceso de industrialización de ese país para hacerlo competitivo frente a los avances de las grandes potencias de ese entonces.

En cuanto a la extensión universitaria los mencionados autores ubican sus inicios en la segunda mitad del S. XIX con los Land Grand Colleges a partir del Morrill - Act (1862), que permitía donar terrenos federales a las universidades públicas y privadas en los Estados Unidos para la promoción del desarrollo agrícola e industrial. A mediados del S. XX se incorporan los conceptos de innovación, emprendimiento, transferencia de tecnología y creación de parques tecnológicos desde las Universidades de Stanford y del Masachusetts Institute of Technology (MIT), pero es solamente hasta la última década del S. XX cuando la Tercera Misión de la Universidad comienza a ser considerada en rigor a partir de los aportes de Etzkowitz y Leydesdorff (1997), reconocidos como los creadores del modelo de la Triple Hélice, fundamentado en los relacionamientos entre las Universidades, el Estado y el Sector Productivo para la generación de valor en un entorno social complejo, basado en el conocimiento. La tercera misión representa, en consecuencia,un conjunto amplio de interacciones de la universidad con la sociedad y es a comienzos del S. XXI cuando la literatura especializada concede al tema un espectro más amplio, como lo recogen Castrillón et al., (2019) a partir de una revisión de la literatura científica sobre Spin Off académicas.

Ndonzuau et al (2002) producto de un análisis comparativo y de la evaluación de los principales problemas evidenciados en ellas durante sus procesos de maduración, proponen un modelo secuencial para poner en vigor Spin Off universitarias desde la generación de ideas de negocio emanadas de dinámicas de investigación e innovación que posibilitan plantear emprendimientos, los cuales, una vez lanzados al mercado en forma de empresas de base tecnológica, se irán fortaleciendo mediante la creación de valor económico. O'Shea et al (2005), con base en un estudio de grupos focales y mediante la aplicación de un modelo econométrico, vinculan atributos de recursos y capacidades, capital institucional, financiero, comercial y humano para indagar porqué unas universidades son más exitosas que otras en la generación de Spin Off, concluyendo que el punto se relaciona en alto grado con la calidad académica y el monto de los fondos destinados por las universidades al fomento de emprendimientos de base tecnológica.

Rasmussen y Borch (2010) a través de un estudio longitudinal proponen un modelo sobre capacidades que facilita a las universidades la conformación de alianzas para la estructuración de Spin Off académicas que considera la creación de una hoja de ruta para la acción, el establecimiento de un equilibrio entre los intereses académicos y comerciales y la incorporación de nuevos recursos, en el marco del ecosistema de base tecnológica que rodea a estas instituciones; Åstebro et al., (2012), tomado como referente un estudio desarrollado en reputadas universidades, demuestran que focalizar las políticas universitarias en emprendimientos liderados por los profesores no es determinante para la puesta en vigor de Spin Off, pues la incidencia de los estudiantes graduados en la generación de este tipo de empresas es mayor que la de sus profesores. Varios estudios dan cuenta de dinámicas acometidas en diferentes países del mundo sobre los relacionamientos universitarios para la puesta en vigor de Spin Off: Kroll y Liefner (2007), a partir de la información aportada por tres universidades de China, concluyen que la flexibilización de las políticas estatales para el apoyo de iniciativas de base tecnológica en ese país genera incrementos significativos en la conformación de Spin Off académicas. Clarysse et al., (2011), con base en un estudio con grupos focales de diversas universidades del Reino Unido aplicado entre 2001 y 2009, encuentran cómo las oportunidades del 
entorno y su base empresarial, combinadas con el nivel académico de las instituciones y la capacidad institucional para el reconocimiento de oportunidades empresariales, son determinantes para que las universidades emprendan mediante Spin Off.

Bathelt et al., (2010), en un estudio de caso despliegan una tipología para caracterización de Spin Off académicas considerando variables como el patrocinio que la institución concede a los interesados; la información disponible para que la institución se involucre con la empresa naciente; las características del conocimiento compartido y la localización de los promotores de la iniciativa. Di Gregorio y Shane (2003) concluyen que la eminencia intelectual, las políticas institucionales de promoción e inversión de recursos en estas iniciativas y privilegiar con regalías conservadoras al inventor dinamizan la creación de Spin Off académicas. Grimaldi et al., (2011), por su parte, en el marco de una reglamentación específica vigente en los Estados Unidos, dan cuenta de la dinámica de emprendimiento académico y la puesta en valor de los resultados de investigación de algunas universidades y de sus impactos en el sector real de la economía, complementando dicho análisis con los requerimientos que sobre el particular reclama la Organización para la Cooperación y el Desarrollo Económico (OCDE) por sus siglas, a sus países miembros, desde una perspectiva sistémica. Rasmussen (2011) a partir de una evaluación de varias instituciones representativas, plantea que conceptos como el ciclo de vida, la teleología, la dialéctica y las dinámicas evolutivas pueden explicar el ciclo de vida de las Spin Off académicas a partir de su estructuración en etapas tempranas, permitiendo una contextualización desde una perspectiva amplia y sistémica. Por su parte Wright (2012) evalúa el papel que las universidades deben jugar en la estructuración de las Spin Off académicas lo micro, en cuanto a sus capacidades y recursos, para asegurar su crecimiento y sostenibilidad y desde lo macro en aspectos de tipo temporal, institucional, social y espacial.

Pazos et al., (2012) con el fin de determinar los factores que influyen en la generación de Spin Off realizan entre 2002 y 2006 un panel con 47 universidades públicas españolas, encontrando que la creación de este tipo de empresas depende en alto grado de la investigación financiada por el sector privado, de la antigüedad de la universidad en este tipo de actividades, de la orientación de la investigación y de la existencia de servicios de incubación al interior de ellas. Por su parte Bigliardi et al., (2013) a partir de una revisión bibliográfica adelantan un estudio tipo Delphi con paneles de expertos para encontrar los indicadores que caracterizan las dinámicas de las Spin Off académicas en Italia y proponen un modelo de evaluación del desempeño de estas organizaciones. Jung y Kim (2018) con la participación de 122 universidades en un estudio realizado entre 2013 y 2015, muestran cómo a partir de la implementación del modelo gubernamental INNOPOLIS Research Institute Spin-off (IRIS), por sus siglas, Corea ha habilitado zonas especiales para que las universidades comercialicen sus resultados de investigación; la evidencia indica que la ubicación de la universidad es determinante frente a las zonas establecidas; los patrocinios financieros del gobierno no son muy representativos y la probabilidad de creación de Spin-off académicas depende de las publicaciones, patentes y fondos de investigación que estos centros educativos provean.

Good et al (2019) proponen un modelo para una estructura organizacional que soporte los ecosistemas de ciencia y tecnología en las universidades; Kalar y Antoncic (2015) encuentran en un estudio desplegado en cuatro universidades de renombre que los estudiantes de programas asociados a las ciencias naturales están mayormente motivados a emprender que los matriculados en ciencias sociales y que no se contraponen los esfuerzos de innovación y transferencia de tecnología en la calidad académica en aquellos programas cuyos docentes promueven el emprendimiento académico; la transferencia de conocimiento ocupa lugar importante en estos procesos. Para Rasmussen et al (2014), el apoyo de los departamentos universitarios a las dinámicas de emprendimiento genera impactos positivos en la estructuración de iniciativas tipo Spin Off; Muscio et al (2016), por su parte, estudian políticas y regulaciones institucionales en Italia encontrando normas que promueven los incentivos monetarios y el riesgo emprendedor, demostrando que los incentivos monetarios tienen alto impacto en emprendimientos de este tipo y que las regulaciones restrictivas los inhiben. SánchezBarrioluengo (2014), plantea que cada institución tiene sus especificidades frente al cumplimiento de su tercera misión y que los modelos estandarizados no aplican. Guerrero et al (2015) estudian los impactos económicos que el fortalecimiento de la investigación y la formación en emprendimiento tienen para la estructuración de iniciativas tipo Spin Off en las universidades.

Ospina (2012) relaciona los factores de éxito y de fracaso asociados a las etapas preliminares a la constitución de organizaciones tipo Spin Off académicas, así: 1) Factores de éxito: a) Cercanía con las Universidades; b) Competencias emprendedoras; c) Entorno favorable; d) Equipo emprendedor; f) Ideas de negocio atractivas en el mercado; g) Capacidad de innovación; h) Características del negocio; i) Obtención capital de riesgo e inversionistas; j) Políticas; k) Existencia de redes; l) Tecnología. 2) Factores de fracaso: a) Ausencia de características emprendedoras; b) Falta de apoyo en la creación de Spin-Off; c) Fracaso comercial del producto; d) Dificultad en el financiamiento; e) Imposibilidad de sostener la capacidad de innovación; f) Obstáculos de gestión y administración; g) Pocas características emprendedoras; h) Problemas financieros; i) Obstáculos relacionados con el mercado; j) Carencia de recursos; k) Apoyo excesivo; I) Ausencia de redes; m) Altas barreras culturales; n) Ausencia de características de universidad investigadora. 


\section{OTROS ANTECEDENTES}

\section{Relación entre universidad- Estado- Sector productivo}

Castillo-Vergara y Alvarez-Marin (2015), a partir del proceso seguido por diversas universidades de Europa y Norteamérica, enfatizan en las dinámicas de transferencia de resultados de investigación que mediante iniciativas tipo Spin Off académicas deberían instaurarse en Chile, recabando en los modelos de negocio y el rol del grupo de investigación, por cuanto, a pesar de su importancia, no han sido suficientemente estimuladas; Monge-Agüero et al., (2015) evalúan el tema del emprendimiento y la estructuración de Spin Off Universitarias en Costa Rica a partir de un estudio exploratorio que caracteriza su surgimiento y sus especificidades; Morales et al (2016) y Zachman y Redchuk (2016), en un estudio basado en entrevistas semiestructuradas a partir de una muestra representativa de Spin Off registradas en el Ministerio de Industria de la Nación, complementado con información secundaria, presentan un trabajo relacionado con la caracterización de las Universidades del Norte de Argentina resaltando su proceso de vinculación con el sector real de la economía a partir de iniciativas tipo Spin Off.

El Observatorio Iberoamericano de la Ciencia, la Tecnología y la Sociedad, OCTE-OEI, (2017) plantea una propuesta metodológica flexible y adaptable a cada institución y sus particularidades con la finalidad de "diseñar, desarrollar e implementar un sistema de indicadores capaces de reflejar la amplia gama de interacciones a través de las cuales las universidades iberoamericanas se relacionan con su entorno"...de manera que esta información permita a los diferentes actores económicos y sociales orientar sus estrategias de búsqueda de vínculos con las universidades. Gomez y Botero (2016) proponen una metodología para el acompañamiento de los actores universitarios implicados en el contexto de un ecosistema de emprendimiento a partir de las fases de proyectos planteadas por el Project Management Institute (PMI por sus siglas) y establece una serie de condiciones mínimas que deberán cumplir las Universidades para acompañar la creación de Spin Off. En lo atinente a estudios sobre Ecosistemas Regionales de Emprendimiento en Colombia, INNPULSA COLOMBIA (Unidad del Gobierno Nacional enfocada en promover el emprendimiento, la innovación y las industrias creativas y culturales como ejes para el desarrollo empresarial y la competitividad del país) y la Universidad Nacional de Colombia sede Medellín (2016), socializan un documento de alta pertinencia en el que se despliega, mediante un mapeo riguroso, una zonificación que comprende las 32 ciudades capitales y diez municipios con experiencias claras en este campo: la capital de Colombia (Bogotá D.C), la Región Norte (Zona del Caribe colombiano), Región Centro Oriente, Región Centro Occidente, Región Sur-Occidente, Región Sur-Oriente y Región Oriente.

Por su parte Rueda-Barrios y Rodenes-Adam (2016), evaluando una muestra de 223 grupos de investigación colombianos registrados y categorizados por el Departamento Administrativo de Ciencia, Tecnología e Innovación (COLCIENCIAS), mediante un tratamiento de carácter empírico (análisis de regresión y ecuaciones estructurales) y conceptualizaciones que incorporan tópicos como la cultura organizacional, la gestión del conocimiento y el capital tecnológico, recogen en una encuesta estructurada aplicada a los líderes de los mencionados grupos frente a sus percepciones sobre la producción científica a partir de "la cultura motivadora, el proceso de externalización en la gestión del conocimiento y el capital tecnológico", concluyendo que estas variables influyen de forma positiva en los resultados obtenidos.

Según Morales et al., (2014), refiriéndose al caso colombiano "La universidad pública presenta diversos obstáculos para poder interactuar de forma eficiente con el sector productivo, algunos de estos relacionados con sus capacidades internas, pero otros por el marco regulatorio que las rige y las condiciones del contexto". Moreno et al., (2014) y Viña et al., (2014), por su parte, dan cuenta de los modelos aplicados por la Escuela de Administración de Negocios (EAN) de Bogotá y de la Universidad Nacional de Colombia, respectivamente, para estos propósitos.

La Asociación Colombiana de Universidades (ASCUN) en un estudio que buscaba referenciar las políticas y prácticas de las universidades colombianas en sus relacionamientos con el sector productivo; Jaramillo (2004); plantea como un imperativo avanzar en el análisis de los procesos administrativos y de las políticas específicas que han utilizado las universidades colombianas y extranjeras para vincularse con su entorno productivo por cuanto, "No es fácil sostener políticas como la construcción de un sistema colombiano de innovación sin universidades cada vez más competentes y sólidas en su gestión de esta vinculación". El ejercicio consideró la aplicación de una encuesta sobre las políticas adoptadas por las universidades colombianas más representativas, tendiente a promover y gestionar sus vínculos de I\&D con empresas públicas y privadas mediante la evaluación de quince características específicas. Los resultados arrojados para la Universidad del Cauca dan cuenta de un panorama alentador. Catorce de los quince puntos de la encuesta demuestran que la institución, en el año 2004, cuenta con indicadores que recogen los requerimientos de los puntos evaluados y, en ese marco, se encuentra en el camino correcto para "promover y gestionar sus vínculos de I\&D con empresas públicas y privadas", al tener claramente definida una visión en tal sentido. En lo que respecta a la creación de nuevas empresas (Spin-Off) derivadas de tecnologías 
desarrolladas en proyectos conjuntos, el documento mencionado plantea: "Este punto no ha sido objeto de discusión profunda hasta el momento y hasta ahora está siendo motivo de reflexión por parte del Consejo de Investigaciones; por lo tanto no hay lugar a desarrollo del mismo."

\section{El marco normativo colombiano}

Si se realiza una evaluación del fenómeno desde la perspectiva constitucional y legal a nivel nación, aunque Colombia ha tenido clara la protección de la propiedad intelectual; el fomento y la protección de la cultura; la importancia de establecer incentivos y estímulos para personas e instituciones que desarrollen y fomenten la ciencia, la tecnología y las demás manifestaciones culturales, así como la libre competencia económica, tal como lo consagran los artículos 61, 70, 71 y 333 de la Carta Política de 1991; expidiendo en 2006 normas de fomento a la actividad emprendedora desde las instituciones educativas (Ley 1014, 26 de enero), es solo hasta el año 2017 cuando el Estado Colombiano, atemperándose a las nuevas exigencias en cuanto a competitividad global y a los compromisos adquiridos en virtud de su adscripción a la Organización para la Cooperación y el Desarrollo Económico (OCDE) por sus siglas, a través del Congreso de la República da cuerpo a diversas normas que realzan el papel de las Universidades y resaltan la transferencia de conocimiento hacia un entorno claramente interesado en poner en valor los resultados generados por los grupos de investigación universitarios.

En efecto, la Ley 1834 de 2017, por medio de la cual se fomenta la Economía Creativa, en su artículo primero consagra como objeto de la misma desarrollar, fomentar, incentivar y proteger las industrias creativas, entendidas estas como aquellas que generan valor en razón de sus bienes o servicios, los cuales se fundamentan en la propiedad intelectual. La Ley 1838 de 2017 por medio de la cual se dictan normas de fomento a la Ciencia, Tecnología e Innovación mediante la creación de empresas de base tecnológica (Spin Off), en su artículo primero plantea como objetivo promover el emprendimiento innovador y de alto valor agregado en las Instituciones de Educación Superior (IES), que propendan por el aprovechamiento de los resultados de investigación y la transferencia de conocimientos a la sociedad como factor de desarrollo humano, científico, cultural y económico a nivel local, regional y nacional, definiendo la Spin Off universitaria como una empresa basada en conocimientos, sobre todo aquellos protegidos por derechos de Propiedad Intelectual, gestados en el ámbito de las Instituciones de Educación Superior (IES), resultado de actividades de investigación y desarrollo realizadas bajo su respaldo, en sus laboratorios e instalaciones o por investigadores a ellas vinculados, entre otras formas. Por su parte la Ley 1923 de 2018 regula lo relativo a los programas y proyectos de inversión que se financiarán con recursos del Fondo de Ciencia, Tecnología e Innovación del Sistema General de Regalías, a través de convocatorias públicas, abiertas y competitivas que se ajusten a los planes y acuerdos estratégicos departamentales en Ciencia, Tecnología e Innovación y a los recursos asignados a cada departamento en el Fondo de Ciencia, Tecnología e Innovación.

Mucha de la información sobre la que se soportó la encuesta aplicada por la Asociación Colombiana de Universidades (ASCUN) para el caso de la Universidad del Cauca referida previamente, fue contextualizada a partir de documentos generados desde la Vicerrectoría de Investigaciones entre 2001 y 2005; lo acaecido durante los años 2006 y 2007 se encuentra consignado en documentos inéditos y no se conocen, hasta la fecha de preparación de este artículo, fuentes de información sistemática que den cuenta cierta de las dinámicas emprendidas por el Sistema de Investigaciones durante los siguientes diez años a partir de 2006, que obren como punto de referencia para la toma de decisiones estratégicas en lo relacionado con la estructuración de un ecosistema de innovación y emprendimiento, con excepción de la normativa generada a partir del año 2017. El Banco Interamericano de Desarrollo (BID), el Departamento Administrativo de Ciencia, Tecnología e Innovación (COLCIENCIAS), el Departamento del Cauca y la Universidad del Cauca recogen los lineamientos del Plan Departamental de Ciencia Tecnología e Innovación para esta región de Colombia, en el que se enfatiza, entre otros aspectos, sobre el desafío que comporta la construcción de sociedades del conocimiento en un ambiente biodiverso de alta heterogeneidad cultural y étnica como el que caracteriza a la región caucana.

Después de trece años de vigencia del Acuerdo 068 de 1998 (Por el cual se crea el Sistema de Investigaciones de la Universidad del Cauca), el 6 de diciembre de 2011 se da luz verde a una nueva norma: el Acuerdo 047 sobre reglamentación del Sistema de Investigación, Desarrollo e Innovación que apunta a fortalecer las actividades de los grupos de I+D y sus relacionamientos con los actores externos, ampliando su campo de acción estratégico. En su artículo cuarto incorpora la difusión, socialización y transferencia de los resultados de las acciones de I+D+i. En el numeral cuatro del artículo quinto, se considera la articulación de los Grupos de Investigación con el entorno local, regional, nacional e internacional. La estructura del Sistema, según el artículo sexto, sufre así algunas transformaciones fundamentadas en la estrategia de articulación de los grupos de investigación con el entorno, en función de las dinámicas de desarrollo e innovación y asume la interacción institucional con el Estado y el sector productivo, dando cuerpo cierto al modelo de la triple hélice, Gutiérrez Ossa (2016). 
Conforme a lo estatuido por el Artículo 48 del Acuerdo 0105 de 1993 por el cual se adopta el estatuto general, se caracteriza a los Centros como "Ia Unidad Interdisciplinaria que se dedica a la investigación científica, a la extensión, a los servicios y a la gestión administrativa"; mediante el Artículo 49, literal 6, de la misma norma se adscriben a la estructura de la Vicerrectoría de Investigaciones tres centros que se consideran estratégicos para el fortalecimiento de esta interacción: Por Acuerdo 005 de 2016 se crea el Centro Internacional para la Investigación del Agua y el Oxígeno, (CINAO); el Acuerdo 053 de 2016 crea el Centro de Innovación y Apropiación Social de la Caficultura, (CICAFICULTURA) y el Acuerdo 054 de 2016 da lugar al Centro Internacional de Secuenciación Genómica y Bioingeniería.

\section{El problema a resolver}

A pesar de lo mencionado, entre 2012 y 2016 las políticas institucionales son poco coherentes al aprobarse el Acuerdo 015 de 2015 por el cual se establece el Sistema de Investigaciones de la Universidad del Cauca que sustituye al Acuerdo 047 de 2011 sobre reglamentación del Sistema de Investigación, Desarrollo e Innovación, incorporando instancias burocráticas poco adecuadas. La estrategia que se soportó en esta estructura nunca pudo concretarse ante el inusitado cambio de Vicerrectores durante un corto lapso de tiempo propiciado por la administración entre los años 2012 y 2017, generando discontinuidad en las políticas; el rol estratégico que se había concedido a los grupos de investigación desaparece, convirtiéndose el proyecto de investigación en el eje nuclear del Sistema por sobre los Grupos de Investigación y los Investigadores que los integran. La mencionada reglamentación plantea una nueva arquitectura organizacional y nuevas funciones para las instancias del Sistema de Investigaciones de la Universidad sin que mediara para ello una prospectiva estratégica rigurosamente gestionada, en abierta contradicción a lo validado por la teoría administrativa en relación a que "La estructura sigue a la estrategia" Chandler (1963). Sin pretender poner en tela de juicio la importancia de los cuerpos colegiados en las decisiones universitarias, es preciso aceptar que el nuevo Consejo de Investigaciones, comparado con el que le precedió, estructurado por méritos y que centraba sus decisiones de manera fluida en el fortalecimiento del grupo de investigación como unidad estratégica del Sistema, se convierte en un organismo burocratizado conformado por once instancias universitarias y una del sector productivo, intrincada estructura muy difícil de convocar, lo cual ha dificultado concretar la estrategia de articulación entre Universidad, Empresa, Estado (UEE).

Si a lo anterior se suma la expedición, en 2016, del Acuerdo 047 por el cual "se ordenan medidas administrativas referentes a la participación de la Universidad en organizaciones de naturaleza pública, privada o mixta", caracterizado por un articulado altamente restrictivo y colmado de consultas ante diez instancias universitarias, lo que implicó inflexibilidades frente al papel que pueden asumir los grupos de investigación en los procesos de articulación con estas organizaciones, sin haber reparado en lo previsto por la legislación colombiana en cuanto al rol que desempeña el sistema educativo en relación con el fortalecimiento del emprendimiento (Ley 1014 de 2006, Artículos 12 y 16), norma que entraría en abierta contradicción con la Ley 1838 de 2017 que da curso a las Spin Off universitarias, se comprende este estado de cosas.

La Universidad del Cauca en el marco del Programa Rectoral propuesto por la nueva administración que asume en 2017 y en cumplimiento de lo inscrito en su Plan de Desarrollo Institucional 2018-2022 (Acuerdo Superior 069 de 2017), en tanto revisa los alcances del Acuerdo 015 de 2015 por el cual se establece el Sistema de Investigaciones, que en su Capítulo I, Artículo 2, numeral 2.4 consagra como uno de sus principales objetivos la difusión, socialización y transferencia de los resultados de las acciones de ciencia, tecnología e innovación a la sociedad, y en su Artículo cuarto, numeral 4.3.4, confiere competencias a la instancia correspondiente (División de Innovación, Emprendimiento y Articulación con el Entorno) para promover y apoyar la vinculación de la investigación que se realiza en la Universidad con el sector productivo, ha concretado, como ya se comentó, reformas sustantivas en su reglamentación interna para atemperarse a estas nuevas realidades con la finalidad de fortalecer sus acciones frente a la estrategia de articulación entre Universidad - Estado - Sector Productivo y Comunidad en cumplimiento de la Tercera Misión.

El Acuerdo 015 de 2015 actualmente se encuentra en proceso de revisión con una metodología soportada en reflexiones rigurosas entre las instancias institucionales competentes y los actores del ecosistema de ciencia y tecnología de la región y el 047 de 2016 fue derogado mediante el Artículo vigésimo del Acuerdo Superior 004 de 2018 por el cual se modifica y adiciona el Acuerdo 08 de 1999 o estatuto sobre la propiedad intelectual, norma que en su Artículo Décimo Octavo formaliza la creación de empresas de base tecnológica (Spin Off) en la Universidad del Cauca.

Este es, en síntesis, el contexto que debe encararse para dar solución a las siguientes preguntas de investigación planteadas a partir de estas reflexiones: ¿Podría catalogarse a la Universidad del Cauca, en tanto Institución pública de Educación Superior del orden nacional, como una Universidad Emprendedora a la luz de la nueva legislación y de las perspectivas mundiales sobre Ciencia, Tecnología e Innovación? ¿Es posible que la institución, en el mediano plazo, en razón de su autonomía, disponga de políticas claras; cuente 
con una apropiada normativa interna sobre propiedad intelectual y con una arquitectura organizacional eficiente que le habiliten para transferir con flexibilidad y poner en valor ante la sociedad, en alianza con inversionistas de talla mundial, el conocimiento generado por sus grupos de investigación, gestando y participando como socia en empresas de base tecnológica tipo Spin Off, sostenibles en los mercados emergentes regionales, nacionales y globales mediante un proceso prospectivo riguroso, soportado en una hoja de ruta pertinente?.

\section{METODOLOGÍA}

(1) Tomando como base las características de mayor relevancia desplegadas por los autores consultados en la literatura especializada que ha sido reseñada, especialmente los factores de éxito y de fracaso relacionados por Ospina (2012) en las etapas previas a la estructuración de Spin Off académicas, se determinan las características clave requeridas para determinar si los grupos de investigación de la Universidad del Cauca reconocidos por COLCIENCIAS en su convocatoria 781 de 2017 poseen condiciones para avanzar hacia la estructuración de iniciativas tipo Spin Off. (2) A partir de esa información se prepara una encuesta compuesta por dieciocho preguntas orientadoras, la cual es sometida a una prueba piloto practicada aleatoriamente entre diez de los grupos que hacen parte del universo censal, de la que se pudo concluir que debían realizarse ajustes de redacción en las preguntas que en nada afectaron su parte sustantiva. (3) Se aplica la encuesta a la totalidad de los 64 grupos de investigación de UNICAUCA reconocidos por COLCIENCIAS EN 2017 mediante un censo. (4) Se tabulan los resultados del censo y de una evaluación de tipo descriptivo se determina, en una primera aproximación, si existen condiciones para que la Universidad del Cauca obre como una Universidad Emprendedora mediante iniciativas tipo Spin Off, alcanzando las metas de articulación y puesta en valor de los resultados de investigación de sus grupos en el entorno local, regional, nacional e internacional. (5) Se despliegan los resultados encontrados en el análisis previo y se emiten las conclusiones correspondientes.

\section{Objetivo General}

Evidenciar las capacidades para poner en operación un Ecosistema de Emprendimiento e Innovación que reafirme las potencialidades de la Universidad del Cauca (UNICAUCA), en tanto Institución pública de Educación Superior del orden nacional, para convertirse, en el mediano plazo, en una universidad emprendedora con iniciativas tipo Spin Off a partir de referentes legales y conceptuales representativos.

\section{Objetivo específico}

Evaluar descriptivamente a partir de los resultados del censo aplicado a los 64 grupos de investigación reconocidos por COLCIENCIAS en 2017, si en el mediano plazo, en razón de su autonomía, la Universidad del Cauca dispondrá de políticas claras y contará con una apropiada normativa interna sobre propiedad intelectual y con una arquitectura organizacional eficiente que le habiliten para transferir con flexibilidad y poner en valor ante la sociedad, en alianza con inversionistas de talla mundial, mediante iniciativas tipo Spin Off, el conocimiento generado por sus grupos de investigación, gestando y participando como socia en empresas de base tecnológica sostenibles en los mercados emergentes regionales, nacionales y globales mediante un proceso prospectivo riguroso, soportado en una hoja de ruta pertinente. Para dar cuerpo a lo planteado se estructuró la encuesta mencionada previamente en dieciocho preguntas orientadoras que se inscriben en la Tabla 1.

Tabla 1: Preguntas orientadoras inscritas en el censo aplicado a los 64 grupos de Investigación de UNICAUCA reconocidos por COLCIENCIAS en la Convocatoria de reconocimiento de grupos de investigación e investigadores 2017

\begin{tabular}{|c|c|}
\hline No & Pregunta orientadora \\
\hline 1 & $\begin{array}{l}\text { ¿Ha conocido usted, directa o indirectamente, el acuerdo } 015 \text { del } 2015 \text { "Establecimiento del Sistema de } \\
\text { Investigaciones de la Universidad del Cauca"? }\end{array}$ \\
\hline 2 & $\begin{array}{l}\text { Si respondió sí en la pregunta anterior, por favor responda a la siguiente pregunta. ¿Considera usted, algún } \\
\text { cambio necesario, (inclusión o exclusión de elementos del acuerdo), dentro del acuerdo para el desarrollo de } \\
\text { proyectos dentro de su grupo de investigación? (Si su respuesta es sí, escriba cuál o cuáles en "otro") }\end{array}$ \\
\hline 3 & ¿Conoce usted la aprobada Ley 1838 de 2017, “Ley de Spin Off”? \\
\hline 4 & $\begin{array}{l}\text { ¿Cree usted que algunas de las investigaciones realizadas o por realizar en su grupo de investigación puedan } \\
\text { resultar en un producto (bien o servicio) encaminado a la solución de alguna problemática local o nacional? }\end{array}$ \\
\hline 5 & $\begin{array}{l}\text { ¿Considera usted que su grupo de investigación ha desarrollado o pueda desarrollar y/o hacer parte de proyectos } \\
\text { con lineamientos de } I+D \text { con base tecnológica? }\end{array}$ \\
\hline 6 & $\begin{array}{l}\text { ¿Considera usted que su perfil como investigador o el de su grupo de investigación puede llevar a desarrollar } \\
\text { iniciativas relacionadas con gestión de emprendimientos de base tecnológica? }\end{array}$ \\
\hline 7 & $\begin{array}{l}\text { ¿Hace parte su grupo de investigación de redes internacionales de alto impacto? (Si su respuesta es sí, escriba } \\
\text { cuál o cuáles en "otro") }\end{array}$ \\
\hline
\end{tabular}


Tabla 1: (Continuación)

\begin{tabular}{|c|c|}
\hline No & Pregunta orientadora \\
\hline 8 & $\begin{array}{l}\text { Si respondió sí en la pregunta anterior, por favor responda a la siguiente pregunta. ¿Alguna de estas redes tiene } \\
\text { experiencia en la generación de Spin Off académicos? }\end{array}$ \\
\hline 9 & $\begin{array}{l}\text { ¿Ha tenido barreras institucionales que hayan impedido o impidan la articulación de la Universidad con actores } \\
\text { externos interesados en aportar recursos y/o capital de riesgo para el desarrollo de iniciativas de base tecnológica } \\
\text { a partir de la producción de los grupos de investigación? (Si su respuesta es sí, escriba cuál o cuáles en "otro") }\end{array}$ \\
\hline 10 & ¿Cómo son los financiamientos de los procesos en su grupo de investigación? \\
\hline 11 & $\begin{array}{l}\text { ¿Ha participado usted o el grupo de investigación en los convenios externos a la universidad para gestionar } \\
\text { emprendimientos? (Si su respuesta es sí, escriba cuál o cuáles en "otro") }\end{array}$ \\
\hline 12 & $\begin{array}{l}\text { Siendo } 1 \text { la menor puntuación y } 5 \text { la máxima, Su interés en participar personalmente o con su grupo de } \\
\text { investigación en convenios interinstitucionales de la Universidad para gestionar emprendimientos de base } \\
\text { tecnológica es: }\end{array}$ \\
\hline 13 & $\begin{array}{l}\text { ¿Cree usted que hay condiciones normativas para que la Universidad del Cauca se convierta, a mediano y largo } \\
\text { plazo, en una Universidad Emprendedora? }\end{array}$ \\
\hline 14 & $\begin{array}{l}\text { ¿Cree usted que hay condiciones locativas y de infraestructura para que la Universidad del Cauca se convierta, } \\
\text { a mediano y largo plazo, en una Universidad Emprendedora? }\end{array}$ \\
\hline 15 & $\begin{array}{l}\text { ¿Cree usted que hay condiciones financieras para que la Universidad del Cauca se convierta, a mediano y largo } \\
\text { plazo, en una Universidad Emprendedora? }\end{array}$ \\
\hline 16 & $\begin{array}{l}\text { ¿Tiene usted y/o su grupo de investigación, relación permanente con otros grupos de investigación? (Si su } \\
\text { respuesta es sí, escriba cuál o cuáles en "otro") }\end{array}$ \\
\hline 17 & $\begin{array}{l}\text { De manera general, ¿Cómo cataloga Ud., la enseñanza y el asesoramiento sobre emprendimiento en los planes } \\
\text { de estudio de las carreras en la Universidad del Cauca? }\end{array}$ \\
\hline 18 & $\begin{array}{l}\text { Ve Ud., en la Facultad de Ciencias Contables, Económicas y Administrativas de la Universidad del Cauca un } \\
\text { punto de apoyo para propiciar el emprendimiento dentro de sus líneas de investigación? }\end{array}$ \\
\hline
\end{tabular}

Se aplicó la encuesta a la totalidad de los 64 grupos de investigación institucionales clasificados por COLCIENCIAS según su categoría, en función de su antigüedad y de la calidad de sus productos de nuevo conocimiento, siendo los grupos A1 los de mayor nivel, seguidos, en su orden, por los grupos tipo A, B, C y R de acuerdo con este criterio de clasificación (Tabla 2):

Tabla 2: Grupos de Investigación de la Universidad del Cauca reconocidos por COLCIENCIAS en la Convocatoria 781 de 2017 según información de la Vicerrectoría de Investigaciones de UNICAUCA 2018

\begin{tabular}{|c|c|c|c|c|c|c|c|}
\hline Unidad académica a la que están adscritos & Grupos & $\mathrm{A} 1$ & A & B & C & $\mathrm{R}$ & $(\%)$ \\
\hline Facultad de Artes & 1 & & & & 1 & & 1.56 \\
\hline Facultad de Ciencias Agrarias & 6 & & 1 & 2 & & 3 & 9.38 \\
\hline $\begin{array}{l}\text { Facultad de Ciencias Contables, Económicas y } \\
\text { Administrativas }\end{array}$ & 7 & & & 2 & 2 & 3 & 10.94 \\
\hline Facultad de Ciencias de la Salud & 8 & & & & 6 & 2 & 12.50 \\
\hline Facultad de Ciencias Humanas y Sociales & 6 & & 3 & & 2 & 1 & 9.38 \\
\hline $\begin{array}{l}\text { Facultad de Ciencias Naturales, Exactas y de la } \\
\text { Educación }\end{array}$ & 22 & 2 & 1 & 6 & 11 & 2 & 34.38 \\
\hline Facultad de Derecho y Ciencias Políticas & 2 & & & & 1 & 1 & 3.13 \\
\hline Facultad de Ingeniería Civil & 1 & & & 1 & & & 1.56 \\
\hline $\begin{array}{l}\text { Facultad de Ingeniería Electrónica y } \\
\text { Telecomunicaciones }\end{array}$ & 7 & 2 & 2 & 1 & 2 & & 10.94 \\
\hline Grupos Inter-institucionales & 4 & 1 & 1 & 2 & & & 6.25 \\
\hline Total Grupos & 64 & 5 & 8 & 14 & 25 & 12 & $100 \%$ \\
\hline Participación porcentual según categoría & $100 \%$ & 7.81 & 12.50 & 21.88 & 39.06 & 18.75 & \\
\hline
\end{tabular}




\section{RESULTADOS}

Tal como se ilustra en la Figura 1 el censo aplicado a los grupos de investigación institucionales reconocidos por COLCIENCIAS en 2017 arrojó los siguientes resultados:

\footnotetext{
¿Tiene usted $y / o$ su grupo de irvestigación, relación permanente con otros grupos de invest igación? (Si su respuesta es si, escriba cuál o cuáles en "otro")

¿Cree usted que hay condiciones financieras para que la Universidad del Cauca se conviert a, a mediano y largo plazo, en una Universidad Emprendedor a?

¿Cree usted que hay condiciones locativas y de infraestructura para que la Universidad del Cauca se convierta, a mediano y largo plaza, en una...

¿Cree usted que hay condiciones normativas para que la Universidad del Cauca se conviert a, a mediano y largo plaso, en una Universidad Emprendedor a?

Siendo 1 la menor puntuación y 5 la maxima, su interès en participar personalmente o con su grupo de investigación en converios...

¿Ha participado usted o el grupo de investigación en las corvenios externos a la universidad para gestionar emprendimientos? (5i su respuesta es si, escriba...

¿Cómo son las financiamientos de las procesos en su grupo de invest igación?

¿Ha terido barreras institucionales que hayan impedido o impidan la articulación de la Univer sidad con actores ext ernos interesados en apor tar recur sos $y / o$.

Si respondió si en la pregunta anterior, por favor responda a la siguiente pregunta 2 Alguna de estas redes tiene experiencia en la generaciōn de Spin Off.

¿Hace parte su grupo de investigación de redes internacionales de alto impacto? (Si su respuesta es si, excriba cuál o cuáles en "otro")

¿Consider a usted que su perfil como investigador o el de su grupo de investigación puede llevar a desarr ollar iniciativas relacionadas con gestión de.

¿Consider a usted que su grupo de investigación ha desarrollado o pueda desarrollar $\gamma / 0$ hacer parte de provectos con lineamientos de I+D con base. ¿Cree usted que algunas de las investigaciones realizadas o por realizar en su grupo de investigación puedan resultar en un producto (bien o servicio)...

¿Conoce usted la aprobada Ley 1838 de 2017, "Ley de Spin Offs"?

Si respondió si en la pregunta anterior, por favor responda a la siguiente pregunta_LConsider a usted, algùn carn bio necesaria, finclusión a exclusión de.

¿Ha conocido usted, directa o indirectarnente, el acuerdo 015 del 2015 "Establecimiento del Sosterna de Investigaciones de la Universidad del Cauca"?
}

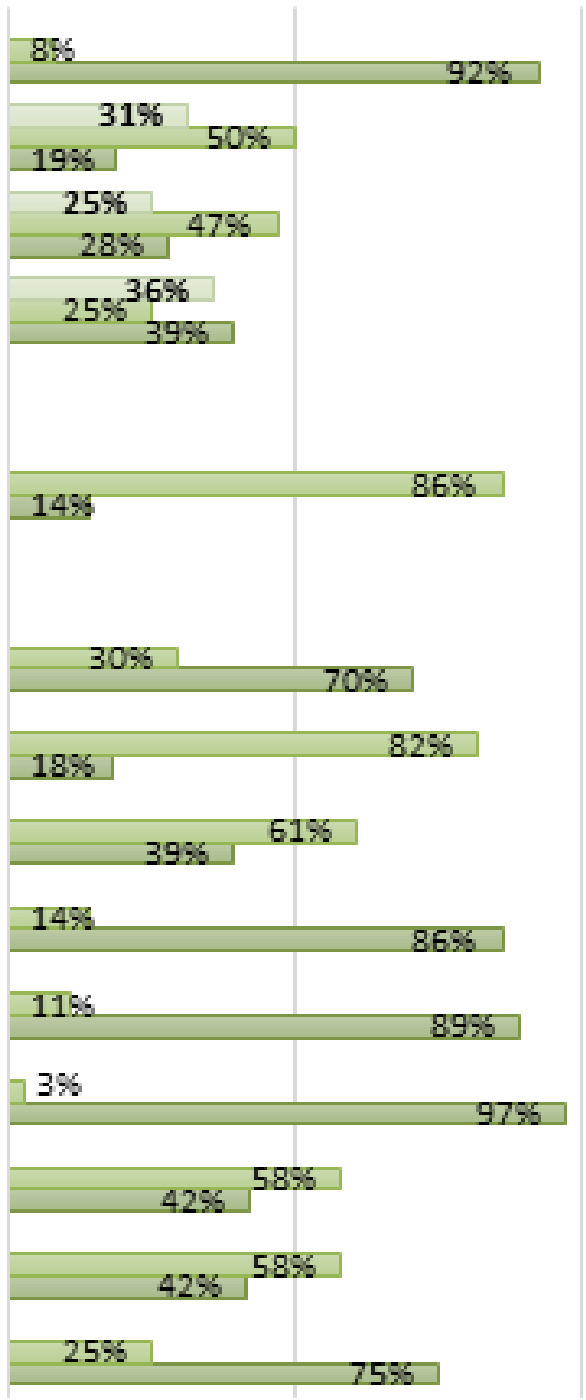

\section{$\neg$ No sabe $\square$ No $\square$ Si}

Fig. 1: Respuestas a preguntas orientadoras de tipo binario en censo aplicado a los 64 grupos de investigación de la Universidad del Cauca reconocidos por COLCIENCIAS en 2017 (\%)

El 75\% (48) de los grupos censados dice conocer el protocolo normativo que regla el sistema de investigaciones de la Universidad del Cauca, en tanto que el 25\%, (16) dicen no conocerlo. Entre los 48 grupos que respondieron afirmativamente a lo anterior, el 41.5\%, (20), consideran oportuno practicar ajustes a la norma, en tanto que 28 no lo han considerado. Los principales ajustes sugeridos son: a) Revisión de la estructura y composición del Consejo de Investigaciones; b) Flexibilización de los trámites administrativos; c) Ampliación de las partidas presupuestales de apoyo a los proyectos de investigación; d) Revisión de la pertinencia de los Comités de Facultad para la Investigación y Posgrados; e) Fusión de los Comités de Ética para la Investigación Científica y Propiedad Intelectual; f) Flexibilización de las políticas de participación de docentes en proyectos de investigación; g) Mayor énfasis en los investigadores y los Grupos de Investigación como unidades centrales del Sistema de Investigaciones; h) Reformulación del Acuerdo 015 como un protocolo de política pues como está estructurado parece más un manual de funciones; i) Precisión del alcance de la División de Articulación con el Entorno (DAE) en cuanto a su rol fundamental como Oficina de Transferencia de Resultados de Investigación. 
El 42\%, (27) de los grupos, dice conocer la Ley 1838 de 2017 por la cual se dictan normas de fomento a la Ciencia, la Tecnología y la Innovación mediante la creación de empresas de base tecnológica (Spin Off) y se dictan otras disposiciones, en tanto que 37 de ellos, (58\%) la desconocen. El 97\%, (62), consideran que sus productos de investigación contribuyen efectivamente a proveer soluciones a problemas locales o nacionales. El 89\%, (57), consideró que, en razón de su perfil y de sus capacidades, ha desarrollado o está en condiciones de desarrollar, o de hacer parte de proyectos de base tecnológica en el contexto de lo aquí considerado. 7 , (11\%) de los grupos de investigación censados, opinaron no estar en condiciones. 39 grupos, (61\%), dicen estar articulados con redes de investigación internacionales de alto impacto, en tanto que los restantes 25 , (39\%), dicen no contar con este tipo de relacionamiento.

El $18 \%$ de los grupos que afirmaron estar articulados con redes internacionales de alto impacto (7), dicen conocer avances tipo Spin Off en ellas; el restante $82 \%$ de ellos (18), no evidencia conocimiento de desarrollos tipo Spin Off en el contexto de sus relacionamientos. El 70\%, (45), dice no haber experimentado barreras institucionales que hayan impedido o impidan la articulación de la Universidad con actores externos interesados en aportar recursos y/o capital de riesgo para el desarrollo de iniciativas de base tecnológica a partir de su producción de conocimiento. Quienes afirman haberlas experimentado, (19), lo atribuyen a las siguientes situaciones: a) Discontinuidad en las políticas entre periodos generan traumas en el desarrollo de los proyectos; b) Reglamentación poco armonizada y carencia de voluntad política para concretar dinámicas de proyectos estratégicos; c) Imposibilidad de cumplir con los cronogramas previstos por inflexibilidades en los tiempos de los trámites; d) Procesos y procedimientos administrativos inflexibles, largos y engorrosos; f) Inflexibilidad en la concesión de tiempos de labor para la investigación a los docentes; g) Desconfianza en las gestiones de investigadores de alto desempeño y reducidos estímulos a su esfuerzo; h) Dificultad para atracción de recursos externos por cobros administrativos onerosos; i) Escasa interacción con actores externos para la dinamización y promoción de proyectos conjuntos; j) Inflexibilidad normativa para la incorporación de donaciones en equipos de alta tecnología por parte de organizaciones cooperantes; k) Excesivos trámites para la gestión financiera y presupuestal de los proyectos; I) La División de Articulación con el entorno no es lo suficientemente efectiva para dinamizar las gestiones requeridas por los grupos de investigación.

Con relación a si existen o no condiciones normativas para que la Universidad del Cauca se convierta, en el mediano y largo plazo en una universidad emprendedora, el 39\%, (25), afirma que sí; el 25\%, (16), que no, y el restante $36 \%$, (23), dicen no saber nada a este respecto. Frente a si existen o no condiciones locativas y de infraestructura, el 28\%, (18), afirman que sí; el 47\%, (30) que no y el restante $25 \%$, (16), dicen no saberlo. En cuanto a si existen o no condiciones financieras, el 19\%, (12), afirma que sí; el 50\%, (32) que no y el restante $31 \%$, (20), dicen no saber nada sobre el particular.

El $92 \%$ de los grupos participantes afirma estar relacionado permanentemente con otros grupos de investigación para adelantar sus dinámicas, en tanto que el $8 \%$ dice no tener ese tipo de relacionamiento.

Frente al rol que podría jugar la Facultad de Ciencias Contables, Económicas y Administrativas de la Universidad como punto de apoyo para propiciar el emprendimiento en las líneas de investigación de los grupos, el 39\%, (25), opinan que sí; el 17\%, (11), que no, y el 28, (44\%), dicen no saberlo. Con relación a las fuentes de financiación de proyectos, la gran mayoría, (55), participan en las convocatorias internas; el 52\%, (33), participa también en convocatorias de COLCIENCIAS; el 27\%, (18), afirma haber participado en convocatorias del Fondo Nacional de Regalías; 15 de ellos, (23\%), dicen haber participado en convocatorias de Cooperación Internacional, 8\%, (5), han establecido relacionamientos de financiación con la empresa privada, $2 \%$, (1) , con el Programa Innpulsa, en tanto que el 32\%, (20), han logrado financiarse con fuentes diversas no consideradas en las categorías precedentes.

En lo relacionado con el interés de participar en convenios interinstitucionales para gestionar emprendimientos de base tecnológica, 5 de los grupos manifestaron poco interés en hacerlo, 11 plantean estar algo interesados en ello, en tanto que 48 dicen tener un grado de interés alto o muy fuerte a este respecto. Desde la perspectiva de la calidad de la formación o asesoría en emprendimiento que se imparte en los diferentes programas académicos por parte de la institución, 43 grupos, (67\%), la consideraron nula o deficiente; $15,(23 \%)$, regular, $6,(9 \%)$, buena y ninguno excelente.

\section{CONCLUSIONES}

De acuerdo con los resultados de este estudio debe resaltarse lo siguiente: 1) La estructura del censo aplicado y las respuestas emitidas por cada uno de los 64 grupos de investigación de UNICAUCA reconocidos por COLCIENCIAS corrobora varias de las condiciones inscritas en la literatura especializada evaluada, para determinar si la institución puede convertirse, en el mediano plazo, en una universidad emprendedora mediante iniciativas tipo Spin Off, a partir de las capacidades de sus grupos de investigación y de las condiciones institucionales. 2) De acuerdo con la información descrita, son más las potencialidades que los 
puntos en contra evidenciados en el marco del análisis planteado. 3) Las condiciones normativas a nivel nacional e institucional a partir de 2017 apuntan a generar capacidades cada vez más importantes relacionadas con la implementación de emprendimientos de base tecnológica tipo Spin Off. 4) La mayoría de los Grupos, el $89 \%$ de ellos, (57), consideró que, en razón de su perfil y de sus capacidades, está en condiciones de desarrollar o de hacer parte de proyectos de base tecnológica mediante iniciativas de tipo Spin Off. 59 de los 64 grupos censados dice estar cohesionado con otros grupos de la Universidad y con Grupos Colombianos de su correspondiente área, dando cuenta de la existencia de sinergias cognitivas suficientes para la gestación de productos de nuevo conocimiento susceptibles de ser transferidos, con valor agregado, hacia el mercado. 5) Es grande el reto de la Facultad de Ciencias Contables, Económicas y Administrativas para fomentar y desplegar dinámicas de capacitación que le posibiliten obrar como un eje de emprendimiento para potenciar las capacidades de los grupos de investigación institucionales; para ello esta unidad académica cuenta en su estructura con la Escuela de Ciencias Contables, Económicas y Administrativas que bien podría asumir el rol que corresponde en este ámbito. 6) Aunque muchos de los grupos de investigación institucionales se encuentran articulados con otros grupos o centros de investigación internacionales de alto impacto, es preciso fortalecer este capital relacional buscando una mayor cohesión global. 7) Se hace necesario intervenir la normatividad interna de la institución, especialmente el Acuerdo 015 de 2015, creando las condiciones requeridas para que la Universidad de Cauca se convierta, a mediano plazo, en una universidad emprendedora mediante la puesta en vigor de Spin Off de base tecnológica, en virtud de los resultados del censo aplicado a los 64 grupos de investigación institucionales en los que se explicitan los brechas que deben ser cubiertas de acuerdo con los resultados del censo aplicado y de lo planteado por la literatura especializada a este respecto.

\section{REFERENCIAS}

Åstebro, T., N. Bazzazian, y S. Braguinsky, Startups by recent University Graduates and their Faculty: Implications for university entrepreneurship policy, https://doi.org/10.1016/j.respol.2012.01.004, Research Policy, 41(4), 663-677 (2012).

Bathelt, H., D. Kogler, y A. Munro, A knowledge-based typology of university spin-offs in the context of regional economic development, Technovation, v. 30 (9-10), pp. 519-532 (2010).

Bigliardi, B., F. Galati y C. Verbano, Evaluating Performance of University Spin-Off Companies: Lessons from Italy, Journal of Technology Management and Innovation, 8(2), 178-188 (2013).

Castillo-Vergara, M., y A. Alvarez-Marin, La transferencia de investigación en instituciones de educación superior mediante spin-off, Revista Actualidades Investigativas en Educación, v. 15, ํㅜ 3, pp. 1-23 (2015).

Castrillón Muñoz., A.J., A. Infante., A. Zúñiga y F.C. Martínez, University Spin-Off: A Literature Review for Their Application in Colombia, https://doi.org/10.14505//jemt.v10.7(33).08, Journal of Environmental Management and Tourism, 10.1(33), 73-86 (2019).

Chandler, A. D., Strategy and Structure: Chapters in the History of the American Industrial Enterprise, 2da Ed., The M.I.T. PRESS 22-199, Cambridge, Massachuseets, USA (1963).

Clarysse, B., V. Tartari, y A. Salter, The impact of Entrepreneurial Capacity, Experience and Organizational Support on Academic Entrepreneurship, https://doi.org/10.1016/j.respol.2011.05.010, Research Policy, 40(8), 1084-1093 (2011).

Di Gregorio, D. y S. Shane, Why do some universities generate more start-ups than others? Research Policy, v.32, n.2, pp.209-227 (2003).

Grimaldi, R., M. Kenney, D. Siegel, y M. Wright, 30 years after Bayh-Dole: Reassessing academic entrepreneurship. Research Policy, v. 40 (8), pp. 1045-1057 (2011).

Good, M., M. Knockaert, B. Soppe y M. Wright, The technology transfer ecosystem in academia. An organizational design perspective, Technovation 82-83, pp. 35-50 (2019).

Gómez Zuluaga, M. E., y J.C., Botero Morales, Startup y Spinoff: Una comparación desde las Etapas para la Creación de Proyectos Empresariales, https://doi.org/10.18566/rces.v24n36.a7, Revista Ciencias Estratégicas, 24(36), 365-378 (2016).

Gutiérrez Ossa, J. A., Modelo de Competencias Investigativas Empresariales desde la Universidad, Empresa y Estado en Colombia, https://doi.org/10.19053/22160159.3771, Praxis \& Saber, 6(12), 241 (2016).

Hernández, C., y D. González, Estudio del Ecosistema de Emprendimiento Tecnológico en Lima: Desafios al 2030. en XVII Congreso Latino-Iberoamericano de Gestión Tecnológica (pp. 1-15), recuperado de http://www.uam.mx/altec2017/pdfs/ALTEC_2017_paper_268.pdf (2017).

INNPULSA COLOMBIA, Universidad Nacional de Colombia sede Medellín, Mapeo e Infografía sobre Ecosistemas Regionales de Emprendimiento en Colombia, Universidad Nacional de Colombia Sede Medellín (2016).

Jaramillo, L.J., Las Políticas Universitarias para la Promoción de Vinculaciones con el Sector Empresarial en I\&D el caso de Colombia: Documento de trabajo, 1-77, Asociación Colombiana de Universidades (ASCUN), Bogotá, Colombia (2005)

Jung, H., y B.K. Kim, Determinant Factors of University Spin-Off: the case of Korea, https://doi.org/10.1007/s10961-0179571-2, 43(6), Journal of Technology Transfer 1631-1646 (2018). 
Kalar B., y B. Antoncic, The entrepreneurial university, academic activities and technology and knowledge transfer in four European countries, Technovation 36-37, pp. 1-11 (2015).

Kroll, H., y I. Liefner, Spin-off enterprises as a means of Technology Commercialisation in a Transforming EconomyEvidence from three Universities in China, https://doi.org/10.1016/j.technovation.2007.05.002, Technovation, 28(5), 298313 (2008).

Morales Rubiano, M. E., P.E. Sanabria Rangel, y P.A Plata Pacheco, Determinantes de la Transferencia de Propiedad Industrial al Sector Productivo en Universidades Públicas Colombianas, https://doi.org/10.25100/cdea.v30i51.43, Cuadernos de Administración, 30(51), 58 (2014).

Monge-Agüero, M., A. Briones y D. Garcia-Perez-de-Lima, Emprendedurismo académico en Costa Rica: un estudio sobre las spin-off académicas, Revista nacional de administración, Vol. 6, no 1, pp. 133-151 (2015).

Morales, M., P. Sanabria y P. Plata, Factores determinantes y tendencias de la transferencia de resultados de investigación universitaria al sector productivo, Criterio libre. Vol. 14, n 25, pp. 135-170 (2016).

Moreno Monsalve., N.A., E.J. Gil Acosta, y F.J. Matiz Bulla, Incidencia del modelo EANTEC \& en la Formación de Emprendedores en Colombia; en Innovación y Emprendimiento a la luz del contexto latinoamericano: Guía de mejores Prácticas para el Impulso de la Actividad Emprendedora basada en la Innovación, Consejo Latinoamericano de Escuelas de Administración - CLADEA, pp 163-176, Lima, Perú (2014)

Muscio, A., D. Quaglione y L. Ramaciotti, The effects of university rules on spin off creation: The case of academia in Italy, Research Policy, 45, pp. 1386-1396 (2016).

Ndonzuau, F. N., F. Pirnay, y B. Surlemont, A Stage Model of Academic Spin-Off Creation, https://doi.org/10.1016/S01664972 (01)00019-0, Technovation, 22(5), 281-289 (2002).

Nunes Gimenez, A. M., y M. B. Machado Bonacelli, Enseñanza superior y sociedad: un estudio exploratorio sobre prácticas de la tercera misión en la Universidade Estadual de Campinas (Unicamp), https://doi.org/10.4067/s071827242018000400094, Journal of Technology Management \& Innovation, 13(4), 94-104 (2018).

Observatorio Iberoamericano de la Ciencia, la Tecnología y la Sociedad (OCTE-OEI); Manual Iberoamericano de Indicadores de Vinculación de la Universidad, con el Entorno Socioeconómico, Red Iberoamericana de Indicadores de Ciencia y Tecnología (2017)

Ospina, N., Éxitos y fracasos en las spin-off académicas. Retrieved from file://C:/Users/000093631/Downloads/spin of universitarias (1).pdf (2012).

O'Shea, R. P., T.J Allen, A. Chevalier y F. Roche, Entrepreneurial Orientation, Technology Transfer and Spinoff Performance of U.S. Universities, https://doi.org/10.1016/j.respol.2005.05.011, Research Policy, 34(7), 994-1009 (2005).

Rasmussen E., S. Mosey y M. Wright, The influence of university departments on the evolution of entrepreneurial competencies in spin-off ventures, Research Policy 43,pp. 92-106 (2014).

Rasmussen, E., Understanding academic entrepreneurship: Exploring the emergence of university spin-off ventures using process theories, International Small Business Journal, 29 (5), pp. 448-471 (2011).

Rasmussen, E. y O.J. Borch, University Capabilities in Facilitating Entrepreneurship: A Longitudinal Study of Spin-off Ventures at Mid-range Universities, https://doi.org/10.1016/j.respol.2010.02.002, Research Policy, 39(5), 602-612 (2010).

Recamán Mejía., A.L. y J.A. Ruiz Gutierrez, Organizaciones Culturales y Creativas: Gerencia e Implicaciones Prácticas, La Salle Ediciones, Ciudad de México, México, 1-230 (2019).

Rodeiro-Pazos, D., S. Fernández-López, L. Otero-González y A. Rodríguez-Sandiás, https://doi.org/10.1016/j.redee.2012.05.006, Resource-based View of University spin-off activity: New evidence from the Spanish case, Revista Europea de Dirección y Economía de La Empresa, 21(3), 255-265 (2012).

Rueda-Barrios, G. y M. Rodenes-Adam, Factores determinantes en la producción científica de los grupos de investigación en Colombia, https://doi.org/10.3989/redc.2016.1.1198, Revista Española de Documentación Científica, 39(1), e118 (2016).

Reyes Valderrabano, B., C. Juárez Landín, M. Martinez Reyes y J.L. Sánchez Ramírez, Modelo de Propiedad Intelectual para Recursos Educativos Abiertos, Campus Virtuales, 6(2), 107-112 (2017).

Sánchez-Barrioluengo, M., Articulating the "three-missions" in Spanish universities, Research Policy, 43, pp. 1760-1773 (2014).

Viña Vizcaíno., A. y G.I. Rodríguez Lozano, Emprendimiento e Innovación Universitarios en Colombia. Una mirada desde la experiencia de la Universidad Nacional de Colombia; en Innovación y Emprendimiento a la luz del contexto latinoamericano: Guía de mejores prácticas para el impulso de la actividad emprendedora basada en la innovación, Consejo Latinoamericano de Escuelas de Administración - CLADEA, pp 177-187, Lima, Perú (2014).

Wright, M., Academic entrepreneurship, technology transfer and society: where next? The Journal of Technology Transfer, v.39 (3), pp. 322-334 (2012).

Zachman P. y A. Redchuk, Singularities of the university spin-off in northern Argentina, en New advances in information systems and technologies, Vol. 2, Part. II, pp. 129-136 (2016). 